\title{
Desarrollo del aparato digestivo en pollos Campero INTA alimentados con poroto mucuna (Stizolobium deeringianum)
}

\author{
Sanz, P.; Revidatti, F.; Fernández, R.; Sindik, M.; Laffont, G. \\ Cátedra Producción de Aves, Facultad de Ciencias Veterinarias, Universidad Nacional del Nordeste, Sargento \\ Cabral 2139, 3400-Corrientes (Argentina). Tel/Fax: 54-379-4425753. E-mail: granja@vet.unne.edu.ar
}

\begin{abstract}
Resumen
Sanz, P.; Revidatti, F.; Fernández, R.; Sindik, M.; Laffont, G.: Desarrollo del aparato digestivo en pollos Campero INTA alimentados con poroto mucuna (Stizolobium deeringianum). Rev. vet. 27: 2, 107-112, 2016. Las leguminosas tropicales se utilizan en los sistemas de producción alternativos para la alimentación de aves. En el presente trabajo se evaluó el efecto de la incorporación de harina de poroto mucuna (Stizolobium deeringianum) en el alimento terminador, sobre el desarrollo del aparato digestivo en pollos Campero INTA. La dieta control se elaboró en base a maíz, soja y una premezcla de vitaminas y minerales; en la dieta de prueba el poroto mucuna reemplazó parcialmente a la harina de soja. El análisis comparativo se realizó mediante ANOVA en un arreglo factorial, operando el sexo y tipo de alimento como efectos principales. No se constataron interacciones significativas entre los distintos niveles de ambos factores para las variables dependientes analizadas. Se constataron diferencias significativas para peso relativo del tracto gastrointestinal con valores de $6,62 \pm 0,62$ y $7,33 \pm 0,59 \%$ para las dietas control y mucuna respectivamente. El peso relativo del estómago muscular registró diferencias estadísticas significativas con valores de $2,56 \pm 0,45$ y $3,06 \pm 0,46 \%$ para las dietas control y mucuna respectivamente. Se demostraron correlaciones positivas y significativas entre el peso relativo del tracto gastrointestinal y del estómago glandular $(r=0,82 ; p=0,01)$ e intestino delgado $(r=0,72 ; p=0,04)$ en el grupo control. En este grupo experimental el estómago glandular correlacionó en forma positiva y significativa con el peso del intestino delgado $(r=0,93 ; p=0,0007)$. En el grupo alimentado con mucuna se registró una correlación positiva y significativa entre el peso relativo del tracto gastrointestinal y del estómago muscular $(r=0,79 ; \mathrm{p}=0,02)$. Se concluye que la incorporación de poroto mucuna produce un incremento en el peso relativo del tracto gastrointestinal. Teniendo en cuenta que dicho poroto podría emplearse en pollos como fuente alternativa de proteínas, se considera necesario profundizar los estudios sobre los métodos de eliminación de sus factores antinutricionales, para una más segura utilización práctica.
\end{abstract}

Palabras clave: pollo, alimentación, poroto mucuna, peso del aparato digestivo.

\begin{abstract}
Sanz, P.; Revidatti, F.; Fernández, R.; Sindik, M.; Laffont, G.: Digestive tract development in Campero INTA chickens fed mucuna beans (Stizolobium deeringianum). Rev. vet. 27: 2, 107-112, 2016. Tropical legumes are used in alternative production systems to feed chickens. We evaluate here the effect of the incorporation of mucuna bean meal (Stizolobium deeringianum) in partial replacement of soybeans in the finisher diet on the development of the digestive tract in Campero INTA chickens. The control diet was developed based on corn, soybeans and a vitamin mineral premix; in the test diet bean mucuna partially replaced soybean meal. The comparative analysis was performed using a factorial ANOVA, with sex and type of food as main effects. No significant interactions between different levels of both factors for the dependent variables analyzed were found. Significant differences for the relative weight of the gastrointestinal tract with values of $6.62 \pm 0.62$ and $7.33 \pm 0.59 \%$ for the control diets and mucuna respectively were found. The relative weight of the gizzard showed statistically significant differences with values of $2.56 \pm 0.45$ and $3.06 \pm 0.46 \%$ for the control diets and mucuna respectively. Significant positive correlations were demonstrated between the relative weight of the gastrointestinal tract and proventriculus $(\mathrm{r}=0.82 ; \mathrm{p}=0.01)$ and small intestine $(r=0.72 ; p=0.04)$ in the control group. In the experimental group the proventriculus
\end{abstract}


correlated positively and significantly with small intestine weight $(r=0.93 ; p=0.0007)$. In the group fed mucuna a positive and significant correlation between the relative weight of the gastrointestinal tract and gizzard $(\mathrm{r}=0.79 ; \mathrm{p}=0.02)$ was recorded. We conclude that the incorporation of mucuna beans in the finisher diet of Campero INTA chickens produces an increase in the relative weight of the gastrointestinal tract, difference that is mainly due to the greater weight of the gizzard. Given that the proximal composition allows mucuna bean consider its use as an alternative source of protein for chickens, it is necessary further studies on methods of removing anti-nutritional factors that enable practical use of such raw materials.

Key words: chicken, feeding, mucuna bean, weight of digestive tract.

\section{INTRODUCCIÓN}

Las familias rurales mantienen distintas especies de aves domésticas con la finalidad del autoconsumo, aunque los pollos dominan la composición de la base animal, pudiendo distinguirse tres sistemas de producción con algunas características comunes. El sistema totalmente extensivo requiere una escasa intervención humana en el ciclo biológico de las aves, las cuales consiguen la mayor parte de su ración diaria a través de la oferta vegetal y animal producida en el ambiente y la obtienen a través de la recolección directa ${ }^{5}$.

En el modelo de traspatio las aves están parcialmente confinadas dentro de un área delimitada o solo tienen acceso a un cobertizo nocturno donde son alimentadas y provistas con agua de bebida. Finalmente en el sistema semi-intensivo el control de los pilares de la producción es más ajustado y los pollos son alimentados con dietas balanceadas producidas por compañías especializadas o con mezclas preparadas a nivel local ${ }^{5}$.

Estos sistemas de producción avícola son fundamentales para garantizar la seguridad alimentaria en los países en vías de desarrollo, debido a que la población rural depende en gran medida de ellos para el consumo de proteína de alto valor biológico. Se ha señalado que la avicultura representa una importante alternativa para alimentar a la población mundial en rápido crecimiento, ya que su producción global ha aumentado un $76 \%$ en los países en desarrollo, estimándose que el sistema de traspatio contribuye en más del $90 \%$ del total de la producción de huevos y carne en los países de bajos ingresos y con déficit de alimentos, situación que probablemente continúe consolidándose en un futuro cercano ${ }^{3}$.

Entre los insumos considerados limitantes de la producción avícola, se destaca el alimento por el alto impacto que el mismo posee sobre el costo total de producción de aves, representando hasta un $70 \%$ del mismo cuando el nivel de inversión es relativamente bajo, situación que se verifica en los sistemas avícolas familiares ${ }^{11}$.

Para fortalecer esta actividad a nivel local y lograr que el potencial productivo existente en las zonas rurales resuelva los problemas alimentarios de la población, se debe tener en cuenta la disponibilidad de materias primas locales para la elaboración de mezclas balanceadas accesibles, para que el pequeño productor rural pueda suministrarlas a sus aves y obtener productos de alto valor nutricional (carne y huevos) de manera consistente a través del tiempo ${ }^{11}$.

La utilización de materias primas alternativas en la alimentación animal tiene por objetivo obtener producciones avícolas eficientes y ecológicamente sostenibles ${ }^{12,15}$. Es necesario incorporar fuentes alternativas de proteína destinadas a elaborar alimentos para los animales, debido al alto costo de la soja, ocasionado por su amplio uso, tanto en alimentación humana y animal, como en biocombustibles ${ }^{9}$.

En las zonas cálidas subdesarrolladas se han ensayado diversas leguminosas tropicales de grano que pueden conformar el componente proteico de las raciones para aves domésticas, debido a que en estas regiones el contenido nitrogenado de las dietas para animales monogástricos constituye el principal factor limitante ${ }^{14}$.

Entre las leguminosas subtropicales, las especies del poroto mucuna se caracterizan por su alto contenido en aminoácidos aunque se ha señalado que la metionina y la cisteína son limitantes en la mayoría de las especies de este género. De particular importancia es su alto contenido en lisina, un atributo nutricional de gran valor que hace de esta leguminosa una importante fuente suplementaria de proteínas ${ }^{1}$. Otro aspecto destacable es la digestibilidad de las semillas, que se sitúa entre 84,2 y $85,5 \%{ }^{1,18}$.

No obstante, estas materias primas contienen factores antinutricionales (FAN), sustancias no fibrosas producidas por el metabolismo de las plantas, como mecanismo de defensa ante el ataque de distintos agentes. $\mathrm{Su}$ presencia en ingredientes utilizados en la alimentación de animales, ejerce efectos contrarios a su óptima utilización, reduciendo el consumo o impidiendo la digestión, absorción y utilización de nutrientes ${ }^{9}$. Los FAN pueden aumentar el peso del aparato digestivo y causar inactivación de enzimas digestivas, disminución de la digestibilidad de proteínas y carbohidratos, reducción de la tasa de crecimiento y alteraciones patológicas en intestino e hígado ${ }^{6,9}$.

El objetivo del presente trabajo fue evaluar el efecto de la incorporación de harina de poroto mucuna (Stizolobium deeringianum), tratado mediante hidratación alcalina, en reemplazo parcial de la soja en el alimento 
terminador, sobre el desarrollo del aparato digestivo en pollos Campero INTA.

\section{MATERIAL Y MÉTODOS}

\section{Animales, instalaciones}

El ensayo se llevó a cabo en el aviario de la Facultad de Ciencias Veterinarias de la UNNE (Corrientes, Argentina). Se dispuso de un galpón semiabierto de 9 x 15 metros, con techo de cinc, paredes de mampostería, extremos cerrados y piso de cemento alisado. Se utilizó un lote de 80 pollos Campero INTA (machos y hembras) de 1 día de edad, provistos por el Instituto Nacional de Tecnología Agropecuaria (INTA EEA Corrientes).

Hasta el día 42, todos los pollos recibieron alimento parrillero iniciador comercial. A partir de esa edad fue posible separar los machos de las hembras basándose en los caracteres sexuales secundarios, lo cual permitió conformar 16 unidades experimentales que fueron alojadas en boxes de $1,5 \mathrm{~m}^{2}$ (8 para machos y 8 para hembras) a razón de 5 aves por box, dentro de los cuales se aplicaron los tratamientos experimentales.

En cada box el agua fue provista con bebederos de plato con recipiente invertido de 4 litros y el alimento con comederos tolva de $3 \mathrm{~kg}$ de capacidad. Durante el período del ensayo se registraron temperaturas máximas con un promedio de $28,8^{\circ} \mathrm{C}$ y mínimas con promedios de $20^{\circ} \mathrm{C}$; la humedad relativa ambiente osciló entre 59 y $86 \%$.

\section{Diseño experimental}

En el presente ensayo se estudió el efecto de la utilización de harina de poroto mucuna (S. deeringianum), en reemplazo de un 30\% de la harina de soja en el alimento terminador (constituido por dos tipos de dietas suministradas ad libitum desde el día 42 al 84 de vida) sobre el desarrollo (peso, longitud) de los órganos del aparato digestivo de pollos Campero INTA, en un arreglo factorial, con el sexo y la dieta como fuentes de variación.

La dieta control se elaboró en base a maíz, soja y una premezcla para alimento balanceado de parrilleros; en la dieta de prueba el poroto mucuna reemplazó parcialmente a la harina de soja. Para la disminución de FAN, el poroto mucuna fue sometido a hidratación alcalina en solución de $\mathrm{Ca}(\mathrm{OH})_{2}$ al 1\% (en relación 1:10 en volumen) durante $24 \mathrm{~h}$ y posterior secado en estufa a $55^{\circ} \mathrm{C}$ durante $48 \mathrm{~h}$ y molido hasta el tamaño de $1 \mathrm{~mm}$.

Las muestras de los ingredientes fueron analizadas mediante el procedimiento de la AOAC (1990) ${ }^{4}$. La composición proximal de la harina de poroto mucuna tratado demostró que contenía $27,56 \%$ de proteína bruta, 3,09\% de extracto etéreo, 5,92\% de fibra bruta y un valor de energía metabolizable de $3.360 \mathrm{kcal} / \mathrm{kg}$.

Cada dieta fue suministrada a 4 boxes de machos y 4 de hembras, constituyendo cada uno una unidad experimental en el ensayo. La composición química de los alimentos se estimó mediante el software para cálculo de raciones Zootec 3.0 y se expone en la Tabla 1 .
Tabla 1. Composición de las dietas control y experimental.

\begin{tabular}{lcc}
\hline composición & control & mucuna \\
\hline maíz (\%) & 65 & 65 \\
soja (\%) & 30 & 21 \\
mucuna (\%) & 0 & 9 \\
premezcla (\%) & 5 & 5 \\
materia seca (\%) & 88,18 & 88,42 \\
proteína bruta (\%) & 17,84 & 16,51 \\
extracto etéreo (\%) & 5,12 & 4,69 \\
fibra bruta (\%) & 3,36 & 3,32 \\
calcio (\%) & 1,09 & 1,08 \\
fósforo (\%) & 0,48 & 0,5 \\
energía metab.(kcal/kg) & 2.936 & 3.013 \\
\hline
\end{tabular}

\section{Variables dependientes}

Al final del ensayo las aves fueron sacrificadas por yugulación previa insensibilización mediante el método aceptado por la Comisión Europea a través de la Directiva 96/609/EEC ${ }^{10}$, tras lo cual se obtuvieron las siguientes variables:

Peso de órganos (\%): se obtuvo dividiendo el peso del aparato digestivo, estómago muscular, estómago glandular, intestino delgado e hígado, por el peso corporal y luego multiplicando por 100 .

Longitud del intestino delgado ( $\mathrm{mm})$ : se midió la longitud del intestino delgado desde su inicio en la unión con el estómago hasta su final, tomando como referencia una perpendicular que pasa por el extremo anterior de los ciegos.

\section{Análisis estadístico}

Se calcularon los estadísticos descriptivos media aritmética, desvío estándar, coeficiente de variación (mínimo y máximo) de cada una de las variables dependientes. La distribución de todas las variables se constató mediante el método de Shapiro-Wilk modificado y la homogeneidad de las varianzas mediante el método de Levene.

El análisis comparativo se realizó mediante ANOVA en un arreglo factorial, tomándose como efectos principales el sexo y tipo de alimento, considerando límite un nivel de significancia del 5\%. Se analizó la interacción sexo por tratamiento con un nivel de significancia del 5\% y las relaciones entre las variables propuestas para cada factor (correlación de Pearson).

\section{RESULTADOS}

En la Tabla 2 se observan los valores de la estadística descriptiva registrados al final del período de estudio.

El resultado del estudio de las interacciones entre los distintos niveles de los factores incluidos en el modelo no fue estadísticamente significativo $(p>0,05)$; ello permitió analizar los efectos principales (dieta y sexo) por separado. 
Tabla 2. Pesos y longitudes finales de los órganos estudiados.

\begin{tabular}{lccccc}
\hline órgano & $\overline{\mathrm{x}}$ & $\mathrm{DE}$ & $\mathrm{CV}$ & mín. & máx. \\
\hline peso tracto gastroint. (\%) & 6,97 & 0,69 & 9,86 & 5,56 & 8,5 \\
peso estómago gland.(\%) & 0,47 & 0,09 & 18,54 & 0,3 & 0,64 \\
peso estómago musc.(\%) & 2,81 & 0,51 & 18,12 & 1,8 & 3,84 \\
peso intestino delg.(\%) & 2,67 & 0,36 & 13,41 & 2,13 & 3,54 \\
longitud intest.delg.(mm) & 139,06 & 16,49 & 11,86 & 117 & 179 \\
peso del hígado (\%) & 1,47 & 0,09 & 6,34 & 1,31 & 1,62 \\
\hline
\end{tabular}

$\overline{\mathrm{X}}$ : media arit., DE: desvío est., $\mathrm{CV}$ : coef.variación, mín: mínimo, máx: máximo.

Tabla 3. Pesos y longitudes finales de los órganos, según la dieta.

\begin{tabular}{lcccccc}
\hline \multicolumn{1}{c}{ órgano } & $\overline{\mathrm{x}}$ control & $\mathrm{DE}$ & $\overline{\mathrm{x}}$ mucuna & $\mathrm{DE}$ & $\mathrm{F}$ & $\mathrm{p}$ \\
\hline peso tracto gastroint.(\%) & 6,62 & 0,62 & 7,33 & 0,59 & 6,43 & 0,03 \\
peso estómago gland.(\%) & 0,45 & 0,1 & 0,5 & 0,07 & 1,74 & 0,21 \\
peso estómago musc.(\%) & 2,56 & 0,45 & 3,06 & 0,46 & 4,9 & 0,04 \\
peso intestino delg. (\%) & 2,68 & 0,42 & 2,67 & 0,31 & 0,01 & 0,93 \\
longitud intest.delg.(mm) & 139,13 & 14,81 & 139 & 19,06 & 0,0003 & 0,99 \\
peso del hígado (\%) & 1,48 & 0,09 & 1,45 & 0,1 & 0,58 & 0,46 \\
\hline
\end{tabular}

$\overline{\mathrm{X}}$ : media aritmética, DE: desvío estándar, F: distribución, p: significancia.

Tabla 4. Pesos y longitudes finales de los órganos, según el sexo.

\begin{tabular}{lcccccc}
\hline órgano & $\overline{\mathrm{x}}$ machos & $\mathrm{DE}$ & $\overline{\mathrm{x}}$ hembras & $\mathrm{DE}$ & $\mathrm{F}$ & $\mathrm{p}$ \\
\hline peso tracto gastroint.(\%) & 6,69 & 0,58 & 7,25 & 0,71 & 4,1 & 0,07 \\
peso estómago gland.(\%) & 0,45 & 0,09 & 0,5 & 0,09 & 0,86 & 0,37 \\
peso estómago musc.(\%) & 2,73 & 0,41 & 2,89 & 0,61 & 0,51 & 0,49 \\
peso intestino delg. (\%) & 2,52 & 0,25 & 2,83 & 0,4 & 2,87 & 0,12 \\
longitud intest.delg.(mm) & 148,9 & 17,77 & 129,25 & 6,82 & 7,64 & 0,02 \\
peso del hígado (\%) & 1,43 & 0,08 & 1,5 & 0,09 & 2,65 & 0,13 \\
\hline
\end{tabular}

X: media aritmética, DE: desvío estándar, F: distribución, p: significancia.

En la tablas 3 y 4 se exponen los pesos y longitudes finales de los órganos del aparato digestivo a los 84 días de edad, para los factores dieta y sexo respectivamente.

En las tablas 5 y 6 se exponen los resultados del análisis de las correlaciones según los tratamientos aplicados. El peso del tracto gastrointestinal correlacionó en forma positiva y significativa con peso del estómago glandular e intestino delgado en el grupo control, mientras que en el alimentado con mucuna la correlación fue positiva pero no significativa. Una situación diferente se observó para la variable peso del

Tabla 5. Análisis de las correlaciones para las variables dependientes del grupo control.

\begin{tabular}{lcccccc}
\hline & PTG & PEG & PEM & PID & LID & PDH \\
\hline PTG & 1 & 0,01 & 0,21 & 0,04 & 0,67 & 0,38 \\
PEG & 0,82 & 1 & 0,94 & 0,0007 & 0,86 & 0,31 \\
PEM & 0,5 & 0,03 & 1 & 0,59 & 0,91 & 0,25 \\
PID & 0,72 & 0,93 & $-0,22$ & 1 & 0,64 & 0,72 \\
LID & $-0,18$ & $-0,07$ & 0,05 & $-0,2$ & 1 & 0,82 \\
PDH & $-0,36$ & $-0,41$ & $-0,46$ & $-0,15$ & $-0,09$ & 1 \\
\hline
\end{tabular}

PTG: peso tracto gastrointestinal, PEG: peso estómago glandular, PEM: peso estómago muscular, PID: peso intestino delgado, LID: longitud intestino delgado, PDH: peso del hígado. tracto gastrointestinal la cual correlacionó en forma positiva y significativa con el peso del estómago muscular en pollos alimentados con la dieta mucuna. Por su parte el estómago glandular correlacionó en forma positiva y altamente significativa con el peso del intestino delgado en el grupo alimentado con la dieta control.

\section{DISCUSIÓN}

Los procesos aplicados para el tratamiento de los nutrientes no tradicionales, particularmente para el poroto mucuna, no se encuentran totalmente ajustados, lo cual origina una marcada variabilidad en su composición y valor nutricional. Los efectos de los procedimientos empleados para la eliminación de FAN pueden implicar mermas en la digestibilidad y absorción intestinal de los nutrientes. La concentración de FAN presentes en el producto final refleja la eficiencia de los procedimientos empleados para su eliminación (remojado en agua, soluciones alcalinas, ácidas, calor y cocción) ${ }^{8}$.

En otros trabajos la inclusión de poroto mucuna en la dieta de pollos produjo incremento del peso relativo del tracto gastrointestinal y aumento de la longitud de intestino delgado, grueso y ciego. Se ha señalado que la presencia de carbohidratos complejos (almidones no digestibles, oligosacáridos y pentosanos) interfieren con la función secretora del aparato digestivo y aumentan la actividad motora de la musculatura lisa del estómago muscular y del intestino delgado, provocando el aumento del tamaño de estos órganos debido al incremento de la viscosidad del contenido gastrointestinal y a la disminución de su tasa de pasaje ${ }^{6,7}$.

Adicionalmente, la incompleta supresión de FAN ejerce acciones perjudiciales sobre los órganos diges-

Tabla 6. Análisis de las correlaciones para las variables dependientes del grupo mucuna.

\begin{tabular}{lcccccc}
\hline & PTG & PEG & PEM & PID & LID & PDH \\
\hline PTG & 1 & 0,06 & 0,02 & 0,07 & 0,25 & 0,46 \\
PEG & 0,68 & 1 & 0,45 & 0,26 & 0,25 & 0,25 \\
PEM & 0,79 & 0,31 & 1 & 0,54 & 0,11 & 0,66 \\
PID & 0,68 & 0,45 & 0,26 & 1 & 0,97 & 0,35 \\
LID & $-0,46$ & $-0,46$ & $-0,61$ & $-0,02$ & 1 & 0,87 \\
PDH & 0,31 & 0,47 & $-0,18$ & 0,38 & $-0,07$ & 1 \\
\hline
\end{tabular}

PTG: peso tracto gastrointestinal, PEG: peso estómago glandular, PEM: peso estómago muscular, PID: peso intestino delgado, LID: longitud intestino delgado, PDH: peso del hígado. 
tivos, generando aumentos de peso y longitud, especialmente en el estómago muscular y el intestino delgado ${ }^{9}$. En el presente trabajo las aves alimentadas con la dieta de prueba registraron un mayor peso del tracto gastrointestinal en general y del estómago muscular en particular, diferencias que fueron estadísticamente significativas. Además, las correlaciones positivas entre los pesos del tracto gastrointestinal y el estómago muscular en este grupo permiten asumir que dicha diferencia se debió fundamentalmente al aumento del tamaño de este órgano en las aves alimentadas con mucuna.

En coincidencia con los hallazgos del presente trabajo, otros investigadores reportaron aumentos del peso relativo del estómago muscular, páncreas e intestino en pollos alimentados con poroto mucuna sin tratar, cambios que fueron revertidos por el tratamiento con calor, lo que apoya la idea de que dicho procesamiento aumenta la digestibilidad de los carbohidratos complejos y disminuye la presencia de FAN en el alimento ${ }^{8}$.

Aplicando distintos métodos de procesamiento del poroto mucuna (humedecimiento, hidratación con hidróxido de potasio y cocción) ${ }^{7}$, se verificó que todos los tratamientos aplicados producen mejoras en el consumo de alimento y en la tasa de crecimiento, con pesos de órganos similares a los controles, concluyendo que el poroto mucuna sin tratar vehiculiza FAN y sustancias amiláceas no digestibles que inducen a un mayor crecimiento de los órganos del aparato digestivo ${ }^{17}$.

Otros reportes señalan que el tratamiento de porotos mucuna con calor húmedo disminuye los efectos de los FAN sobre los órganos, haciendo posible su inclusión como sustituto del poroto de soja en dietas para pollos de carne. En dicho trabajo se incorporó harina de semillas de poroto mucuna hervido a $100^{\circ} \mathrm{C}$ durante 90 minutos, a concentraciones de 5, 10, 15 y $20 \%$ en dietas de terminación de pollos parrilleros, observándose que el peso de los órganos del aparato digestivo no fue estadísticamente diferente, lo cual sugiere que la presencia residual de FAN no posee efectos demostrables sobre los órganos del aparato digestivo ${ }^{2}$.

El factor sexo no influyó sobre el peso relativo de los órganos del aparato digestivo en los pollos del presente estudio, en tanto que la longitud del intestino delgado registró valores más altos en machos que en hembras, diferencias que fueron estadísticamente significativas. Otros autores compararon híbridos de crecimiento lento del genofondo cubano de pollos para carne versus híbridos de rápido crecimiento, incluyendo ambos sexos en un diseño factorial (genotipo*sexo) y -en discrepancia con los resultados aquí obtenidos- encontraron diferencias significativas en los indicadores técnicos de la producción a favor de los machos, mientras que el peso de vísceras fue mayor para las hembras independientemente del genotipo al que pertenecieran ${ }^{19}$.

Estudiando los efectos de la interacción genotipo*sexo sobre las características de la carcasa y desarrollo de órganos del aparato digestivo en pollos para carne, incluyendo las estirpes comerciales Mar- shall, Arbor Acres y Hubbard, se demostraron valores similares para el peso del estómago muscular y ausencia de interacciones genotipo*sexo en las poblaciones estudiadas ${ }^{16}$.

Comparando los pesos de estómago muscular e hígado de tres variedades de la raza indígena africana Tswana (variedades cuello desnudo, normal y enana) incluyendo aves de ambos sexos en un diseño experimental factorial con el genotipo y sexo como fuente de variación, se reportó una interacción genotipo*sexo para el peso del estómago muscular. En las aves portadoras del gen de cuello desnudo los machos registraron mayor peso del estómago muscular en tanto que en pollos de emplume normal y en los portadores del gen del enanismo, no existieron diferencias para este carácter ${ }^{13}$.

En conclusión, la incorporación de un $9 \%$ de harina de porotos mucuna tratados con solución alcalina de hidróxido de calcio al 1\%, genera indeseables incrementos de peso y longitud del tracto gastrointestinal en pollos Campero INTA. Teniendo en cuenta que la composición proximal del poroto mucuna habilita su uso como fuente alternativa de proteínas en monogástricos, se considera necesario profundizar los estudios sobre los métodos de eliminación de FAN para posibilitar su utilización en nutrición animal.

\section{REFERENCIAS}

1. Adebowale YA, Adeyemi I.A, Oshodi AA, Niranjan K. 2007. Isolation, fractionation and characterisation of proteins from Mucuna bean. Food Chem 104: 287-299.

2. Akinmutimi A, Okwu N. 2006. Effect of quantitative substitution of cooked mucuna utilis seed meal for soybean meal in broiler finisher diet. Int J Poultry Sci 5: 477-481.

3. Alabi RA, Esobhawan AO, Aruna MB. 2006. Econometric determination of contribution of family poultry to women's income in niger-delta, Nigeria. J Centr Europ Agric 7: 753-760.

4. AOAC. 1990. Official Methods of Analysis, 15th ed., Publ. Association of Official Analytical Chemists, Virginia, USA.

5. Besbes B. 2009. Genotype evaluation and breeding of poultry for performance under sub-optimal village condition. World's Poultry Sci J 65: 260-271.

6. Bressani R. 1993. Grain quality of common beans beans. Food Rev Int 9: 217-297.

7. Carew LB, Hardy D, Weis J, Alster F, Mischler SA, Gernat A, Zakrzewska EI. 2003. Heating raw velvet beans (Mucuna pruriens) reverses some anti-nutritional effects on organ growth, blood chemistry, and organ histology in growing chickens. Trop \& Subtr Agroecosyst $J 1$ : 267-275.

8. Carew L, Gernat A. 2006. Use of velvet beans, Mucuna spp., as a feed ingredient for poultry: a review. World's Poul Sci J 62: 131-144.

9. Chaparro SP, Aristizábal ID, Gil JH. 2009. Composición y factores antinutricionales de las semillas del género Mucuna. Rev Fac Nal Agr Medellín 62: 4843-4853. 
10. Close B, Banister K, Baumans V, et al. 1996. Recommendations for euthanasia of experimental animals: Part 1. Lab Anim 30: 293-316. Disponible en: www.fao.org/ag/ esp/revista/0203sp1.htm

11. FAO. 2002. Avicultura familiar. Rev Avicult Prof 20: 16-17.

12. González C, Diaz I, Vecchionacce H, Novoa L. 1999. Potencialidad de la producción de cerdos a campo en Venezuela. VEncuentro Nutr y Aliment Monogástr. http://www. sian.info.v/porcinos/publicaciones/encuentros/gonzalezeivone.htm

13. Kgwatalala PM, Bolowe AM, Thutwa K, Nsoso SJ. 2013. Carcass traits of the naked-neck, dwarf and normal strains of indigenous Tswana chickens under an intensive management system. Agric Biol J N Am 4: 413-418.

14. Lon-Wo E, Cino D. 2000. Aminoácidos sintéticos en la eficiencia de utilización de una fuente proteica (Vigna unguiculata) alternativa para pollos de ceba. Rev Cub Cien Agric 34: 341-346.

15. Nieves D, Dilcia BA, Pineda A, Silva L. 2001. Digestibilidad in vivo de nutrientes en dietas para conejos con niveles crecientes de naranjillo (Thrichantera gigantea). Anales XVI Reunión de la Asociación Latinoamericana de Producción Animal (ALPA), La Habana, Cuba.
16. Olawumi SO, Fajemilehin SO, Fagbuaro SS. 2012. Genotype $\times$ sex interaction effects on carcass traits of three strains of commercial broiler chickens. $J$ World's Poult Res 2: 21-24.

17. Tuleun CD, Offia B, Yaakugh ID. 2010. Comparative performance of broiler fed diets containing raw and processed Mucuna seed meal. Int J Poult Sci 9: 1056:1062.

18. Vadivel V, Janardhanan K. 2005. Nutritional and antinutritional characteristics of seven south Indian wild legumes. Plant Foods Hum Nutr 60: 69-75.

19. Valdivié M, Sampedro J, Cabezas L, Pérez N, Dieppa O. 2001. Comparación de híbridos nacionales e importados de pollos de engorde en Cuba. Crianza en jaulas en el verano. Rev Cub Cienc Agríc 35: 257-261.

\section{Revista Veterinaria ingresa a SciELO}

Revista Veterinaria, publicación oficial de la Facultad de Ciencias Veterinarias de la Universidad Nacional del Nordeste (Corrientes, Argentina), ha logrado acceder al Núcleo Básico de Revistas Científicas Argentinas (Nivel 1), luego de calificar adecuadamente en el Centro Argentino de Información Científica y Tecnológica (CAICYT), según Resolución 2485/14 del Consejo Nacional de Investigaciones Científicas y Técnicas (CONICET).

Sobre un puntaje máximo de 33 se obtuvieron 32 puntos. Tal calificación constituye "una garantía de la excelencia de la publicación" (sic) y queda expedita la vía del Portal SciELO (Scientific Electronic Library Online) para los artículos publicados.

En tal calificación gravitó positivamente la circunstancia de haber aumentado el índice de impacto (Scimago-Elsevier) y haber disminuido las autocitaciones. También se tuvieron en cuenta aspectos como la amplia cobertura de la revista, la calidad científica del Comité Editorial, los criterios de evaluación de los artículos, el origen de los autores (locales $60 \%$, nacionales $13 \%$, extranjeros $26 \%$, en idioma inglés), el adecuado balance entre trabajos científicos originales y reseñas bibliográficas (ambos con alta calidad), así como el estricto cumplimiento de la periodicidad semestral y la favorable acogida por indizadores como Cab, Doaj, Ebsco, Gale Cengage, Infocyt, Latindex y Scopus.

Se consolida de esta manera la continuidad de "Revista Veterinaria", que en su acontecer supera 50 años de existencia en nuestra Facultad, la cual en 2016 cumplió el $96^{\circ}$ aniversario de su fundación. 